\title{
The Effects of Brain Stem Transections on the Neuronal Networks Responsible for Rhythmical Jaw Muscle Activity in the Guinea Pig
}

\author{
Scott H. Chandler and Michael Tal ${ }^{1}$ \\ Department of Kinesiology and the Brain Research Institute, University of California, Los Angeles, \\ Los Angeles, California 90024
}

\begin{abstract}
The purpose of this study was to determine the critical areas of brain stem necessary for the production of cortically induced rhythmical jaw muscle activity (RJMs) in the anesthetized guinea pig. It was found that longitudinal midline transections of the lower brain stem starting at the obex and extending to the rostral third of the inferior olivary nucleus (IO) were able to abolish rhythmical EMG activity in the jaw-opener muscle (digastric) on the same side (ipsilateral) as the cortical stimulus. Under these conditions, rhythmical activity in the contralateral digastric (DIG) EMG was not affected. Midline transections extending from the rostral superior colliculus to $500 \mu \mathrm{m}$ rostral to the trigeminal motor nucleus had no effect on cortically evoked bilateral DIG rhythmical EMG activity. Serial transverse hemisections of the left side of the medulla, starting at the obex and extending to the rostral third of the IO, reduced the amplitude of the left DIG EMG without producing significant effects in the cycle characteristics [cycle duration (CD) or burst duration (BD)] of that muscle during stimulation of the contralateral (right) cortex. Hemisections more rostral to the IO completely abolished bilateral rhythmical DIG activity induced by stimulation of the cortex on the side opposite to the transection (right cortex). Under these conditions, stimulation of the cortex on the side ipsilateral to the left hemisection (left cortex) initiated rhythmical EMG activity with normal cycle characteristics in only the contralateral (right) DIG muscle. Transverse hemisections as little as $500 \mu \mathrm{m}$ rostral to the trigeminal motor nucleus (Mot V), which spared the pyramidal tract, had no effect on RJMs induced by stimulation of either cortex. These data suggest that (1) each ipsilateral cortex initiates activity in neuronal oscillatory networks located exclusively in the contralateral brain stem; (2) bilateral rhythmical DIG activity is produced by neurons in the contralateral brain stem; (3) each side of the lower brain stem is capable of producing rhythmical DIG activity independent of the integrity of the other side; and (4) the location of the neuronal oscillators responsible for RJMs is between the rostral IO and the trigeminal motor nucleus.
\end{abstract}

It has been known for some time that repetitive stimulation of the masticatory cortex in a variety of animal species can initiate rhythmical jaw movements (RJMs) that resemble mastication (see Dubner et al., 1978; Luschei and Goldberg, 1981, for reviews). These jaw movements were shown to consist of rhythmi-

Received Aug. 10, 1985; revised Dec. 6, 1985; accepted Dec. 10, 1985.

This research was supported by National Institute of Dental Research Grant DE 06193. We would like to thank Naomi Goffman for her fine histological assistance, and we would also like to thank the anonymous reviewers of the manuscript for their excellent suggestions for improvement.

Correspondence should be addressed to Scott H. Chandler, Ph.D., Department of Kinesiology, UCLA, 2851 Slichter Hall, 405 Hilgard Avenue, Los Angeles, CA 90024.

' Dr. Tal was on sabbatical leave from the Hebrew University, Department of Anatomy, School of Dentistry, Jerusalem, Israel.

Copyright @ 1986 Society for Neuroscience $0270-6474 / 86 / 061831-12 \$ 02.00 / 0$ cal activity in jaw-opener (digastric) muscles. It was subsequently demonstrated that these RJMs are not critically dependent on peripheral feedback for the genesis of the basic oscillatory pattern (Chandler and Goldberg, 1982; Dellow and Lund, 1971; Nakamura et al., 1976), but are produced by a central neural network commonly referred to as a central pattern generator (CPG). The importance of reflex inputs for the proper coordination of jaw movements during RJMs has been studied extensively (Goodwin and Luschei, 1974; Lund and Dellow, 1971; Lund et al., 1981; Sumi, 1977; see Dubner et al., 1978; Lund and Olsson, 1983; Luschei and Goldberg, 1981, for a review). However, little attention has been given to the identification or location of the critical elements that comprise the neuronal networks responsible for the oscillation. Transection studies have shown that the brain stem caudal to the diencephalon is capable of generating elementary forms of RJMs (Bremer, 1923; Grill and Norgren, 1978; Lund and Dellow, 1971; Thexton et al., 1980). The exact rostral-caudal boundaries of that portion of the brain stem critical for generating RJMs have not been systematically investigated. Anatomical studies in the cat (Mizuno et al., 1983) and rat (Travers and Norgren, 1983; Vornov and Sutin, 1983) have shown that pre-motoneurons projecting to the trigeminal motor nucleus (Mot V) are bilaterally distributed throughout a large region of lateral reticular formation (RF) in the pons and medulla. Electrophysiological experiments have identified cells in the medial reticular formation of the lower brain stem that are rhythmically active during chewing in the cat (Nakamura et al., 1980, 1982b, 1984). The extent to which these cells are responsible for jaw oscillation has not been determined.

A related issue with respect to the location of the brain stem areas essential for RJM production is whether each side of the brain stem contains neuronal networks capable of producing oscillatory activity independent of the contralateral side. Given that the mandible is fused at the midline, the nervous system must produce bilateral jaw muscle synergies during RJMs. It could be hypothesized that a single oscillatory network in the brain stem is responsible for the fundamental bilateral rhythmical oscillation of the jaw during RJMs. In fact, most models for RJM circuitries have depicted the neuronal oscillator network as a single entity that imposes bilateral rhythmical activity in the appropriate jaw-opener and jaw-closer motoneurons (Chandler and Goldberg, 1982; Murakami et al., 1980; Nakamura et al., 1979; Sumi, 1971). However, the questions of how the cortex initiates bilateral rhythmical activity in the appropriate masticatory motoneurons and the extent to which oscillation on one side is dependent on oscillation of the other have not been addressed.

The purpose of the present study was to elucidate, with the use of selective transection techniques, both the location and organization of brain stem neuronal network(s) and pathways responsible for mediating rhythmical activity in both the left 
and right digastric (jaw-opener) muscles during stimulation of the masticatory cortex in the guinea pig.

\section{Materials and Methods}

Experiments were performed on 31 albino guinea pigs weighing 400$600 \mathrm{gm}$. Thirty minutes prior to administration of the anesthetic agent, the animals were given Thorazine (chlorpromazine hydrochloride, 12.5 $\mathrm{mg} / \mathrm{kg}, \mathrm{i} . \mathrm{m}$.) and atropine sulfate $(0.1 \mathrm{mg} / \mathrm{kg}$, i.p.). The animals were then anesthetized with ketamine $\mathrm{HCl}(110 \mathrm{mg} / \mathrm{kg}$, i.m. $)$. Dexamethasone $(0.5-1.0 \mathrm{mg}$, i.m.) was given at the start of each experiment to reduce possible brain stem swelling as a result of the transections. A standard tracheotomy and jugular vein cannulation were performed. Bipolar recording EMG clectrodes $(0.13 \mathrm{~mm}$ outer diameter; tip exposure, 2.0 $\mathrm{mm}$ ) were inserted in both the left and right anterior belly of the digastric (DIG) muscle. In a previous publication (Goldberg et al., 1982), it was shown that there is a precise correspondence between the activity in the DIG muscle and the resulting jaw-opening movement. In the present experiments jaw movements were not monitored with a tracking device, but when rhythmical DIG activity was present, jaw movements were observed. Fine wire bipolar electrodes were also inserted into the left oral mucosal area to induce the jaw-opening reflex (Chandler and Goldberg, 1982; Sumino, 1971). The animals were then placed in a standard Kopf stereotaxic device, and a circular area of skull overlying the masticatory cortex on both sides of the midline was removed. A bipolar stimulating electrode (Rhodes, SNE 200) was inserted into both the left and right masticatory cortex to induce RJMs. All open wound edges wcre infiltrated with $2 \%$ lidocaine every $2 \mathrm{hr}$. The details of both the surgical procedures and the method of identification of the masticatory region of the cortex have been described previously (Chandler and Goldberg, 1982; Goldberg et al., 1982; Lambert et al., 1985).

To perform brain stem transections of the medulla and pons $(n=$ 14), the skull overlying the cerebellum was removed, and the surface of the brain stem was exposed by gentle aspiration of the overlying cerebellum. For transections or electrolytic lesions of the midbrain $(n=$ 17), the occipital cortex was removed, exposing the underlying tectum. Spontaneous respiration was assisted by artificial ventilation at a rate of $40-45$ strokes $/ \mathrm{sec}$ of $5-10 \mathrm{~cm}^{3} / \mathrm{stroke}$ of room air. Expired $\mathrm{CO}_{2}$ was monitored with a Beckman LB-2 gas analyzer and maintained to within 3-5\%. Under these conditions, cortically induced RJMs were easily obtained for many hours. Data collection usually began $1 \mathrm{hr}$ after the removal of the cerebellum or occipital cortex.

Both midline transections and transverse hemisections of the left brain stem were carried out with the use of either an adjustable fine wire knife (Kopf) or a fine razor blade (1.5 mm wide) attached to a stereotaxic holder. The Kopf knife consists essentially of a stainless steel cannula assembly containing a movable fine wire. The protrusion of this wire from the tip of the cannula can be adjusted to a maximum of $2.5 \mathrm{~mm}$. Once protruded, the wire bends approximately $90^{\circ}$, forming a hook. This assembly, when attached to the Kopf manipulator, can be stereotaxically lowered into the brain stem, and the wire can then be protruded from the cannula tip. With the use of the manipulator the wire can be raised through the brain stem the desired amount, thereby transecting the neural tissue. This procedure allows for very accurate and fine control of brain stem transections with a minimal amount of bleeding. Several transections were usually made in a given experiment. Data were obtained beginning $1 / 2$ to $1 \mathrm{hr}$ after a given transection.

Electrolytic lesions were performed by passing $500 \mu \mathrm{A}$ anodal DC current for $30 \mathrm{sec}$ through one pole of a bipolar strut electrode (Kopf, SNE 200). This produced lesions approximately $1 \mathrm{~mm}$ in diameter, as verified by subsequent histological examination.

To activate the short-latency polysynaptic cortico-trigeminal pathways without simultaneous activation of the neuronal networks responsible for oscillation of the jaw, short pulse train (SPT) stimulation of the cortex was elicited. The cortical stimulation consisted of a SPT stimulus to the left or right masticatory cortex (4 pulses, $500 \mathrm{~Hz}, 0.5$ $\mathrm{msec}, 1 / \mathrm{sec}$ ) between 1.5 and $2 \times$ threshold for evoking the contralateral DIG EMG response (in the text, ipsilateral and contralateral DIG responses always refer to the side of the cortical stimulus). Thresholds were determincd immediately prior to the initial transection. The mean amplitudes of 16-32 consecutive ipsilateral and contralateral DIG responses to left and right SPT stimulation were determined with the use of a signal averager (Dagan, 4 channel) triggered by the initial cortical stimulus pulse.

The RJMs were induced by $10-15 \mathrm{sec}$ of repetitive stimulation of the cortex at $40 \mathrm{~Hz}$. The intensity was adjusted to $2-3 \times$ threshold (thresholds usually $50-200 \mu \mathrm{A}$ ) for evoking rhythmical digastric EMG activity prior to the initial transection. Subsequent to the transection, a response was considered abolished if no intensity was capable of initiating activity in that muscle. In early experiments, if a response was abolished, the cortical electrode position was changed to determine if the lack of response was a function of cortical position. In no case was this found to bc truc. Thereforc, in most experiments the cortical electrode position was not changed after a transection. The precise relationship between left and right DIG EMG activity during RJMs before and after a transection was determined by a signal-averaging program on an IBM-XT laboratory computer. The reference event (trigger) was the contralateral DIG EMG, and its onset is depicted by a vertical line in all figures. Individual cycle durations (CD), burst durations (BD), and the value of integrated EMG (mV-sec) activity of rectified DIG EMG of at least 20 consecutive cycles of EMG activity were also calculated by computer. Means and SD were then calculated. Programs were developed by RC Electronics (Santa Barbara, CA). To determine if the lesions significantly affected $(p \leq 0.05)$ cycle characteristics for a given animal, Student's $t$ tests were performed on the control versus transected conditions. In some cases, a repeated measures, block design analysis of variance (Danicl, 1983) was performed when comparisons between and within subjects, or between successive transections in the same animal, were desired.

All anatomical measurements were referenced to the obex. Supplemental doses of ketamine $(25-50 \mathrm{mg})$ were given intravenously every $2 \mathrm{hr}$ or if any signs of spontaneous limb movements were present. Under these conditions, the animal was not responsive to pinching of the pad of either foot or pinna and would lie motionless except when exhibiting RJMs. During RJMs the only visible movements were those of the mandible. At the end of each experiment the animal was sacrificed, and the brain was removed and fixed in formalin. Serial sections, $50 \mu \mathrm{m}$ thick and stained for Nissl substance with carbol fuchsin, were obtained for histological verification of the locations and extent of the transections or lesions.

\section{Results}

\section{Midline transections}

We have previously shown that stimulation of either the left or right masticatory cortex with a SPT (4 pulses, $500 \mathrm{~Hz}, 0.5 \mathrm{msec}$ ) produces a short-latency (8-11 msec) bilateral activation of the DIG muscles (Fig. 1, $A, C$ ), while repetitive stimulation of the same cortical site at $40 \mathrm{~Hz}$ produces RJMs of 3-5 Hz concomitant with bilaterally synchronous EMG activity in both DIG muscles (Fig. 2, $A, B$ ) (Lambert et al., 1985). To determine the importance of the continuity of both sides of the lower brain stem on both the bilateral DIG responses produced by SPT stimulation of the cortex and the ability of the cortex to initiate RJMs, a midline transection starting at the obex was performed. The results from a representative animal are shown in Figures 1 and 2. Figure 1 shows the effects of a midline transection on the left and right DIG responses induced by SPT stimulation of the cortex. The control DIG responses to SPT stimulation of the left cortex are shown in Figure $1 A$, while the control mean responses to right cortical SPT stimulation are shown in Figure $1 C$. It was observed that a midline transection extending from the obex to the rostral third of the inferior olivary nucleus (IO) and nucleus ambiguus blocked the ipsilateral digastric EMG response to SPT stimulation at any current intensity (Fig. $1 B$, upper trace; Fig. $1 D$, lower trace). During these conditions, the mean contralateral DIG EMG response to the cortical stimulus remained close to control amplitude (compare contralateral control responses of Fig. 1, $A$ and $C$, with contralateral responses after transection in Fig. $1, B$ and $D$ ). Figure 2 shows the effects of this same midline transection on cortically induced RJMs. Figure 2, $A$ and $B$, shows the averaged relationship between the left and right DIG EMG induced by either left $(A)$ or right $(B)$ repetitive cortical stimulation prior to a midline transection. Figure 2, $C$ and $D$, shows the averaged relationship between 


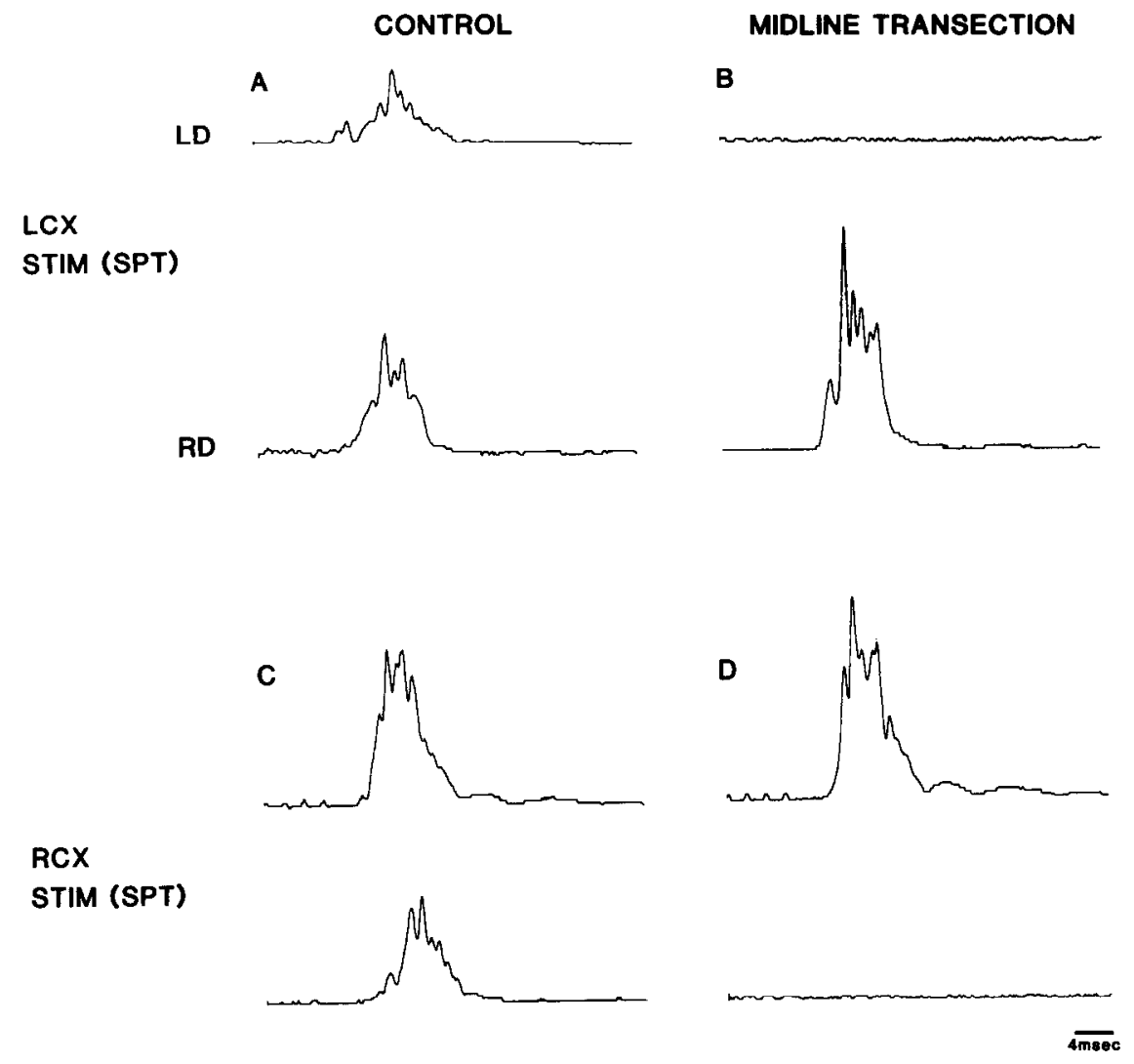

Figure 1. Effect of a midline transection of the medulla on the left and right digastric (DIG) response induced by short pulse train stimulation (SPI) of the masticatory cortex. Control left $(L D)$ and right $(R D)$ DIG responses evoked by stimulation $(S T I M)$ of the left $(L C X)$ or right $(R C X)$ cortex are shown in $A$ and $C$, respectively. The responses evoked after a midline transection of the medulla extending from the obex $3.5 \mathrm{~mm}$ rostral to the level of the rostral third of the IO and nucleus ambiguus are shown in $B$ and $D$. Note that, for stimulation of either the left or right cortex, the response in the ipsilateral DIG was abolished. Cortical stimulation: 3 pulses, $500 \mathrm{~Hz}, 0.5 \mathrm{msec}$. In all subsequent figures, $R D$ and $L D$ refer to right and left DIG muscles, and LCX and $\mathrm{RCX}$ refer to the side of the cortical stimulation. both DIG muscles after a series of midline transections that extended from the obex to the rostral IO. It can be seen that, regardless of the side of cortex stimulated, the ipsilateral DIG EMG response was abolished while the contralateral averaged response was minimally affected. Transections placed as little as $500 \mu \mathrm{m}$ caudal to the effective transection for blocking the ipsilateral digastric EMG response generally reduced the amplitude of the ipsilateral DIG response without affecting the basic oscillation observed in the EMG. The rostral extent of the midline transection is shown in the cross section in Figure $3 \mathrm{~A}$.

It was observed that a midline transection, sufficient to abolish the ipsilateral DIG response during both SPT and repetitive cortical stimulation, had no significant effects on the CD or BD of the contralateral DIG EMG during RJMs induced by repetitive cortical stimulation. This is shown in the bar graph of Figure $2 E$ and further confirmed by signal averaging of the left and right DIG EMGs during RJMs induced by stimulation of the left or right cortex (Fig. $2, A-D$ ). These data suggest that the cortex does not directly activate ipsilateral DIG motoneurons during RJMs through ipsilateral corticobulbar pathways, but in fact must activate relay neurons in the contralateral brain stem, which then recross the midline in the medulla before projecting to the ipsilateral DIG motoneuronal pool. Furthermore, it is obvious that the continuity between both sides of the lower brain stem at the rostral IO is not essential for oscillation to occur in the contralateral DIG EMG (Fig. 2, $C, D$ ).

The above data suggest that the initial level of decussation of the corticobulbar fibers is rostral to the level at which the premotoneuronal fibers recross the midline. To determine at what level the corticobulbar fibers cross the midline, and to confirm our previous observation that the ipsilateral cortex does not directly project to ipsilateral DIG pre-motoneurons, midline transections of the midbrain and rostral pons were performed. Midline transections of the midbrain had little effect on the rhythmical digastric activity during repetitive stimulation of the cortex. Figure 4 presents data from an animal that had undergone a series of midline transections starting at the rostral superior colliculus and extending caudally to the level of the Mot V. When the transection extended to approximately $500 \mu \mathrm{m}$ rostral to the motor nucleus, both the cycle durations and burst durations of DIG EMG activity were only modestly affected (Fig. $4 B$ ). Figure $4 C$ shows the mean $C D$ and $B D$ of the contralateral (right) DIG EMG during RJMs induccd by lcft cortical stimulation prior to (dark box) and after (hatched box) a midline transection starting at the superior colliculus and extending caudally to approximately $500 \mu \mathrm{m}$ rostral to the Mot V. Although there was a small $(<10 \%)$ but significant change in burst duration $(p \leq 0.05)$, there was no significant effect on cycle durations after the transection. When the lesion extended more caudal than the Mot V, the ability to induce RJMs on either side by cortical stimulation was abolished. This indicates that the corticobulbar fibers responsible for initiation of RJMs decussate at the level of the motor nucleus of $\mathrm{V}$. Furthermore, these data demonstrate that the ipsilateral corticobulbar fibers do not initiate RJMs, since, if they did, the upper brain stem midline transections would not have blocked RJMs bilaterally.

\section{Transverse hemisections of the brain stem}

In order to determine the area of lower brain stem necessary for the production of cortically induced RJMs, serial transverse hemisections of the left brain stem, starting at the obex, were performed. Figure 5 shows representative data from one animal that had undergone a series of serial transections starting at the obex and proceding rostrally. In this example, only data from two of the more rostral transections are shown, since the more caudal hemisections had no effect on RJMs. The averaged relationships between both DIG muscles to repetitive stimulation of the left or of the right cortex prior to serial hemisections are 

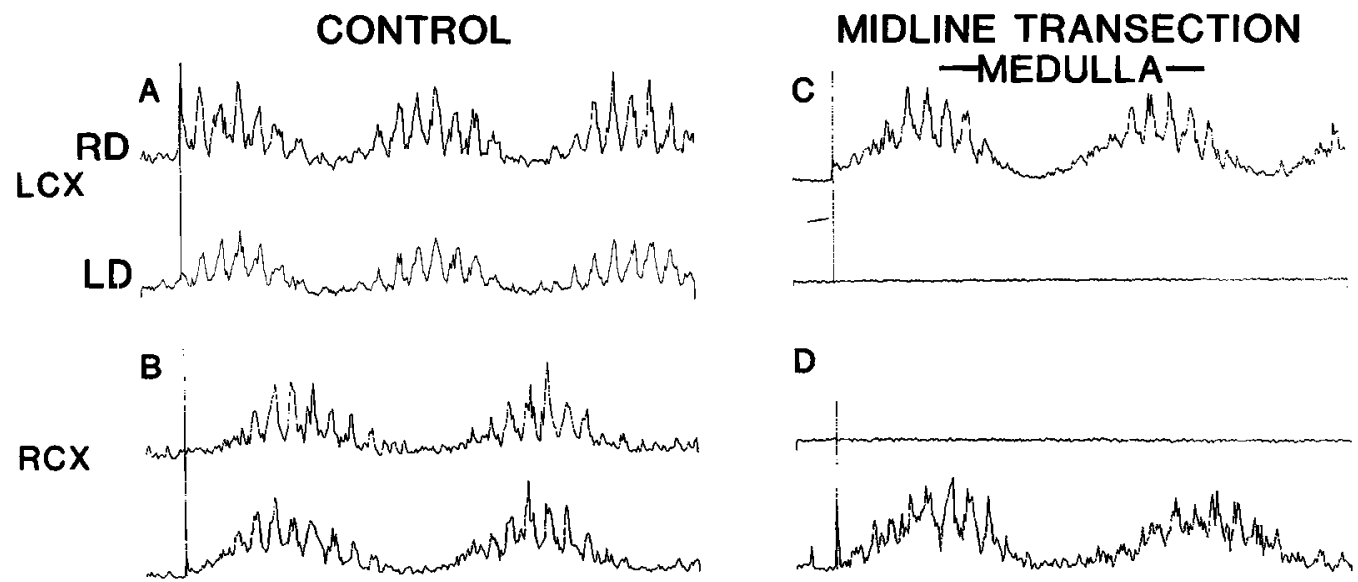

D

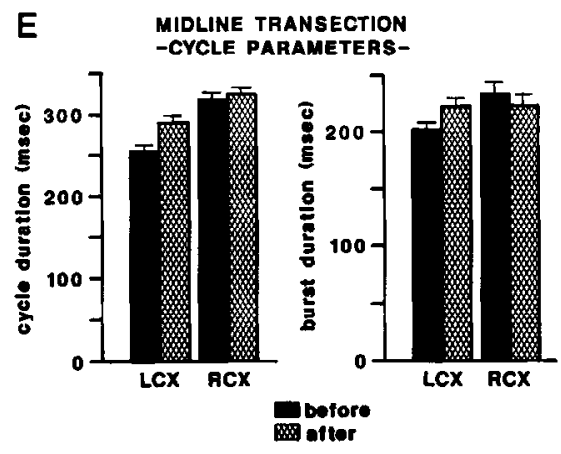

Figure 2. Effect of a midline transection of the medulla on the averaged left and right DIG EMG activity during rhythmic jaw muscle activity (RJMs) induced by repetitive cortical stimulation. $A$, Signal average of left and right DIG EMG was determined by 25 consecutive RJMs cycles. The reference (trigger) event was always the DIG EMG on the side contralateral to the cortical stimulus and is represented by the vertical line in each trace. The threshold voltage for the trigger was set at the onset of the DIG EMG. $A$ and $B$ show the control EMG relationships between left and right DIG muscles during stimulation of the left $(A)$ or right $(B)$ cortex. In $C$ and $D$, the EMG relationships between both muscles after a midline transection extending from the obex to the rostral third of the IO are shown. The data in this figure were taken from the same animal represented in Figure 1. Time calibration in $D, 100 \mathrm{msec} . E$, Mean CDs and BDs for the 25 consecutive cycles of contralateral DIG activity during RJMs, taken before (solid bars) and after (hatched bars) the transection. Vertical lines, Standard deviations. Student's $t$ tests showed no statistical difference between the control and transection conditions for either CD or BD.

shown in Figure 5, $A$ and $B$, respectively. The averaged EMG responses resulting from a left transverse hemisection, placed $500 \mu \mathrm{m}$ caudal to the effective hemisection for complete abolition of RJMs induced by stimulation of the right cortex, are shown in Figure 5, $C$ and $D$. It was observed that during stimulation of the right cortex, the activity in the ipsilateral (right) DIG EMG (Fig. $5 D$, upper trace) was abolished, while the amplitude of the contralateral (left) DIG EMG was reduced (Fig. $5 D$, lower trace). Furthermore, as shown in the bar graph of Figure $5 F$, no. 3 , the mean cycle duration of the contralateral (left) DIG EMG was not statistically different from control. Stimulation of the left cortex under these conditions produced oscillation in both DIG muscles (Fig. $5 C$ ). The mean CD for the contralateral (right) DIG was also not statistically changed (Fig. $5 F$, no. 3). Figure $5 E$ shows the averaged EMG response to stimulation of the left cortex in the same animal after a hemisection was placed $500 \mu \mathrm{m}$ more rostral than the previous one. After this hemisection, the ipsilateral (left) DIG EMG response was markedly reduced (Fig. $5 E$, lower trace), while the amplitude and oscillation were still present at near control levels in the contralateral (right) DIG EMG (Fig. $5 E$, upper trace). The mean $\mathrm{CD}$ of the contralateral (right) EMG was not statistically different from control (Fig. $5 F$, no. 4). Under these conditions, stimulation of the right cortex was not capable of producing RJMs (not shown). Figure $3, A$ and $B$, shows the level of neuraxis in which a left transverse hemisection is sufficient to abolish RJMs induced by repetitive stimulation of the right cortex.

In general, although the amplitude of the DIG EMG was reduced with each more rostrally placed hemisection during RJMs induced by stimulation of the right cortex, the fundamental RJM CD and variability remained relatively constant prior to complete abolition (Fig. $5 F$; compare control with nos. 1-4).

Table 1 summarizes the cycle characteristics for three animals before and after left hemisections approximately $0.5 \mathrm{~mm}$ caudal to the critical transection for abolition of RJMs induced by right cortical stimulation. A block-design analysis of variance for the three animals showed that a left transverse hemisection placed just caudal to the critical lesion for RJM abolition significantly reduced (approximately $40 \%$ ) the value of the contralateral (left) integrated DIG EMG (I-EMG), while producing only a modest reduction $(12 \%)$ in the mean CDs. In addition, there was no significant change in the BDs of the DIG EMG activity. In one animal, a complete bilateral transverse transection of the brain stem was performed. It was observed that the location of the critical transection for complete abolition of RJMs was similar to that observed for the hemisected animals. Furthermore, there were no significant changes in mean CDs or BDs before $(\mathrm{CD}=$ $277 \pm 30 \mathrm{msec}, \mathrm{BD}=153 \pm 39 \mathrm{msec}$ ) or after a complete transection placed approximately $500 \mu \mathrm{m}$ caudal to the critical transection for RJM abolition ( $\mathrm{CD}=273 \pm 45 \mathrm{msec}, \mathrm{BD}=$ 


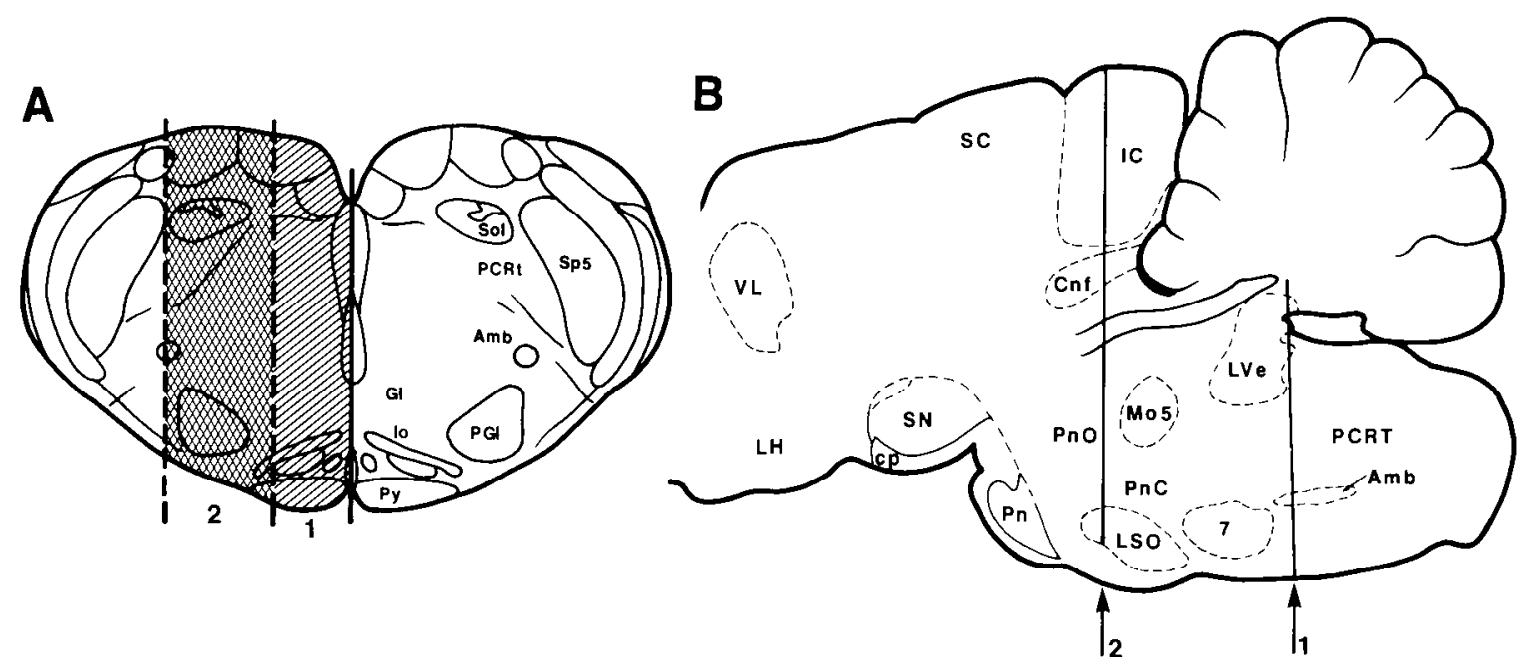

Figure 3. A, Transverse section of the medulla approximately $3.5 \mathrm{~mm}$ rostral to the obex at the rostral third of the IO and nucleus ambiguus. Midline transections separating the left and right brain stem extending from the obex to this level of brain stem completely abolished ipsilateral DIG activity induced by cortical stimulation. In another series of experiments (see text), transverse hemisections of the left brain stem were performed. Shaded area on left brain stem section, Lateral extent of the transverse hemisections of the brain stem. Here, 1 refers to hemisections that extended approximately $1 \mathrm{~mm}$ lateral to midline, while 2 refers to hemisections that extended from midline to approximately 2 mm lateral from midline. $B$, Sagittal section of brain stem taken $2 \mathrm{~mm}$ lateral from midline. Vertical lines, Rostral-caudal boundaries of brain stem that must remain in continuity for cortically induced RJMs to occur. Transverse hemisections caudal to $I$ had minimal effects on rhythmical DIG activity induced by right cortical stimulation. Lesions at the level of 1 abolished RJMs induced by right cortical stimulation. Transverse hemisections of the left brain stem rostral to the level shown in 2 (sparing corticobulbar fibers) had minimal effects on RJMs induced by left or right cortical stimulation. Hemisections just caudal to the level shown in 2 abolished RJMs induced by stimulation of the left cortex, but had no effect on RJMs induced by stimulation of the right cortex. See text for a detailed description of experiment. $A m b$. Ambiguus nucleus; $C n f$, cuneiform nucleus; $c p$, cerebral peduncle; $G i$, gigantocellular reticular nucleus; $I C$, inferior colliculus; $I o$, inferior olive; $L H$, lateral hypothalamus; $L S O$, lateral superior olive; $L V e$, lateral vestibular nucleus; $M o 5$, trigeminal motor nucleus; $P C R t$, parvocellular reticular nucleus; $P G i$, paragigantocellular reticular nucleus; $P n$, pontine nucleus; $P n C$, pontine reticular nucleus caudalis; $P n O$, pontine reticular nucleus oralis; $P y$, pyramidal tract; $S C$, superior colliculus; $S N$, substantia nigra; $S o l$, solitary nucleus; $S p 5$, spinal trigeminal nucleus; $V L$, ventrolateral thalamus; 7 , facial nucleus.

$143 \pm 39 \mathrm{msec}$ ). Given that these results were similar to those obtained for the left hemisection experiments, additional bilateral transections were not performed.

Figure 6 gives an example of the ipsilateral and contralateral
DIG response to repetitive stimulation of the left or right cortex in another animal, before and after two hemisections of the left brain stem placed at the level of the rostral pole of the IO (3.5 $\mathrm{mm}$ rostral to the obex). The level of transection is shown in

\section{CONTROL}

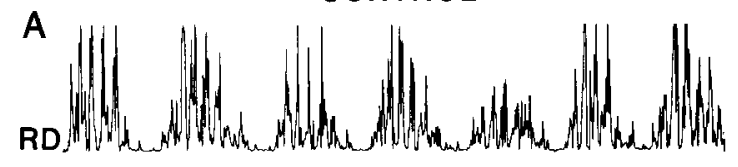

LCX

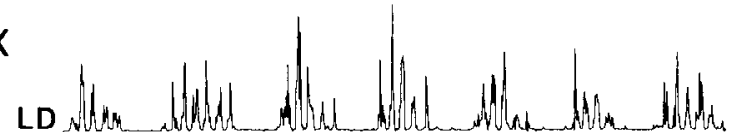

MIDLINE TRANSECTION -MIDBRAIN-

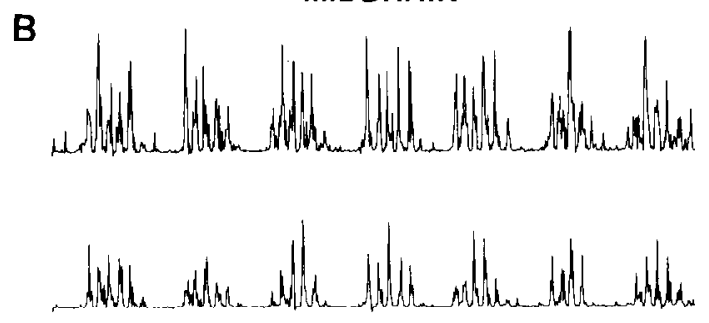

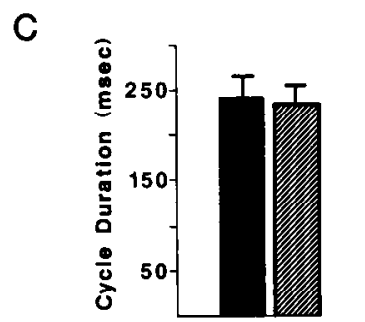

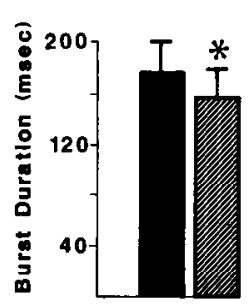

Figure 4. Effect of a midline transection of the midbrain and rostral pons on DIG EMG activity during RJMs induced by repetitive stimulation of the left cortex. $A$, Left and right DIG EMG responses induced by stimulation of the left cortex before transection. $B$, EMG responses after a midline transection extending from the rostral superior colliculus to approximately $500 \mu \mathrm{m}$ rostral to the motor nucleus of $\mathrm{V}$ (Mot V). Time calibration, $B, 200 \mathrm{msec}$. C, Mean CDs and BDs of the right DIG (contralateral) EMG before (solid bars) and after (hatched bars) the transection. Calculations based on 25 consecutive DIG EMG cycles during RJMs. Mean BD showed a slight $(<10 \%)$ but significant $(p<0.5)$ decrease after the transection. 
Figure 5. Averaged relationship between left and right DIG before and after serial transverse hemisections of left brain stem. $A$ and $B$, Signal average of left and right DIG EMG activity during RJMs $(n=30)$ induced by stimulation of either the left $(A)$ or right $(B)$ cortex prior to hemisections. The reference event (trigger) was the onset of the contralateral DIG EMG, represented as a vertical line in each trace. $C$ and $D$, Averaged EMG data after a series of hemisections extending to $3 \mathrm{~mm}$ rostral to the obex. Note that the averaged cycle duration is similar to control $(A, B) . E$, Averaged EMG data induced by stimulation of the left cortex after the hemisection extended another $500 \mu \mathrm{m}$ rostral. At this time, RJMs were not elicited by stimulation of the right cortex. Time calibration, $E, 150$ msec. $F$, Mean cycle durations of the contralateral DIG EMG before and after four serial hemisections of the left brain stem, starting at the obex. Hemisections 1 , 2. and 3 , separated by $1 \mathrm{~mm}$, while distance between 3 and 4 is $500 \mu \mathrm{m}$. Means were determined by 30 consecutive RJM cycles. Vertical lines, Standard deviations. Repeated measures analysis of variance showed no significant differences for cycle durations between transections.

\section{LEFT HEMISECTION}

LCX STIM
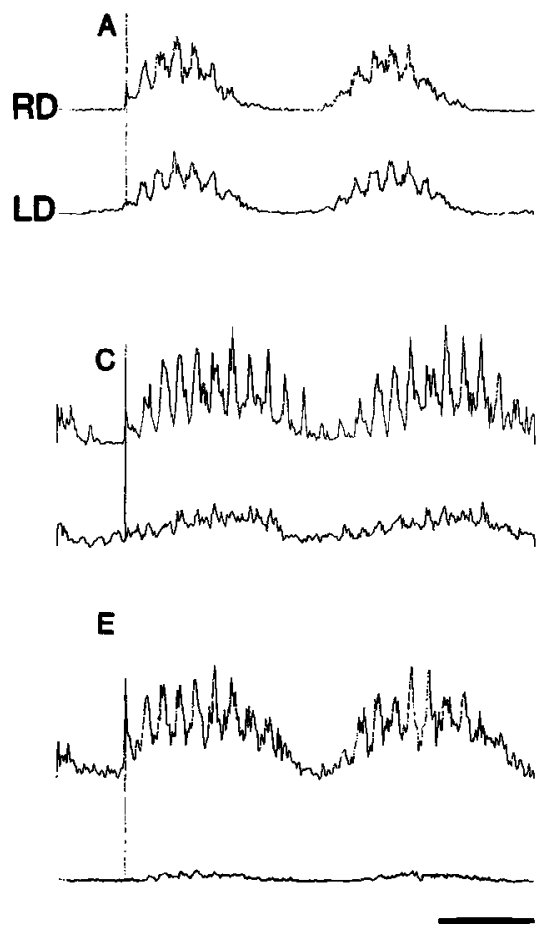

RCX STIM

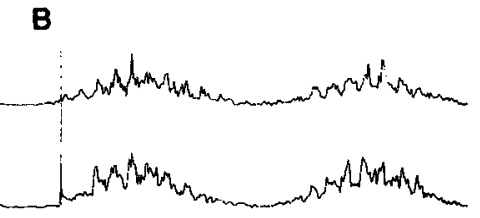

the cross section in Figure $3 A$. The first transection extended $1.5 \mathrm{~mm}$ lateral to the midline (Fig. $3 A$, no. 1 ), while the second extended $2 \mathrm{~mm}$ lateral to the midline (Fig. $3 A$, no. 2). As compared to the control DIG responses (Fig. 6, $A, B$ ), the transection that extended $2 \mathrm{~mm}$ lateral to the midline abolished the RJMs induced by stimulation of the right cortex (Fig. $6 F$ ), while stimulation of the left cortex induced rhythmical activity in only the contralateral (right) DIG (Fig. 6E, upper trace). It was observed that the transection extending only $1.5 \mathrm{~mm}$ lateral to midline did not abolish rhythmical DIG activity (Fig. 6D). Although the interburst periods showed increased baseline activity and the amplitudes were somewhat reduced, the RJM pattern was still observed in the ipsi- and contralateral DIG EMG during stimulation of the right cortex (Fig. 6D). At this level of transection, stimulation of the left cortex induced activity only in the contralateral (right) DIG EMG (Fig. 6C, upper trace).

The spinal trigeminal nucleus is known to contain interneurons that mediate the reflex excitation of digastric motoneurons during the jaw-opening response induced by peripheral stimulation of the oral mucosa (Sumino, 1971). In order to rule out the possibility that the effects of brain stem hemisections on the DIG motoneurons were due to interruption of the fibers originating from this nucleus that project to DIG motoneurons and receive cortical input (Kuypers, 1958; Zimmerman et al., 1964),

Table 1. Effects of a left hemisection on cycle parameters

\begin{tabular}{|c|c|c|c|c|c|c|}
\hline & \multicolumn{2}{|c|}{$\mathrm{CD}(\mathrm{msec} \pm \mathrm{SD})$} & \multicolumn{2}{|c|}{$\mathrm{BD}(\mathrm{msec} \pm \mathrm{SD})$} & \multicolumn{2}{|c|}{ I-EMG $(m V-\sec \pm S D)$} \\
\hline & Control & Lesion & Control & Lesion & Control & Lesion \\
\hline \multicolumn{7}{|l|}{ GP1 } \\
\hline$(n=20)$ & $350 \pm 43$ & $293 \pm 35$ & $161 \pm 61$ & $163 \pm 41$ & $26.2 \pm 11$ & $17.6 \pm 6$ \\
\hline \multicolumn{7}{|l|}{ GP2 } \\
\hline$(n-20)$ & $347 \pm 44$ & $316 \pm 34$ & $236 \pm 47$ & $240 \pm 27$ & $41.6 \pm 10$ & $24.0 \pm 5$ \\
\hline \multicolumn{7}{|l|}{ GP3 } \\
\hline$(n=20)$ & $268 \pm 53$ & $239 \pm 21$ & $137 \pm 51$ & $125 \pm 18$ & $13.3 \pm 6$ & $8.6+4$ \\
\hline \multirow[t]{2}{*}{ Change (\%) } & \multicolumn{2}{|c|}{$12^{a}$} & \multicolumn{2}{|c|}{1} & \multicolumn{2}{|c|}{$38^{a}$} \\
\hline & \multicolumn{2}{|c|}{$F_{21}=28.5$} & \multicolumn{2}{|c|}{$F_{2,1}=0.08$} & \multicolumn{2}{|c|}{$F_{2,1}=70.6$} \\
\hline
\end{tabular}

Cycle parameters were calculated in three guinea pigs (GP1-3) after a transverse hemisection of the left side of the brain stem $500 \mu \mathrm{m}$ caudal to the effective hemisection for complete abolition of RJMs. Data for each animal before and after the hemisection were obtained from 20 consecutive left DIG EMG cycles during RJMs induced by stimulation of the right cortex. Means \pm SD are given. Abbreviations: Cycle duration (CD), burst duration (BD), and integrated EMG (IEMG).

${ }^{a} p \leq 0.001$. 


\section{LEFT HEMISECTION}

\section{LCX STIM}
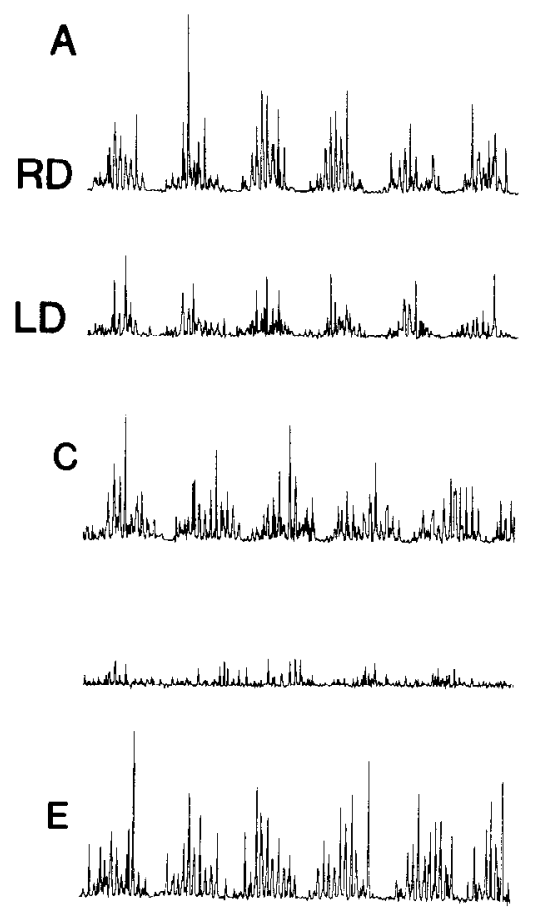

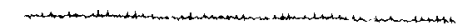

B
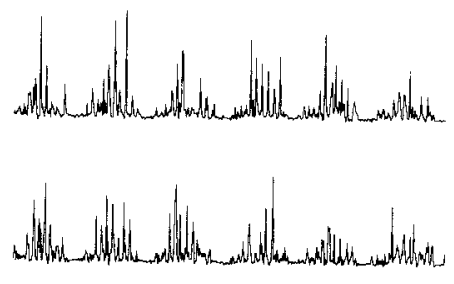

D
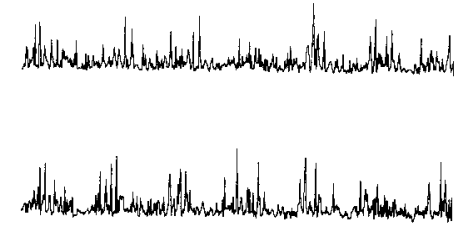

$F$

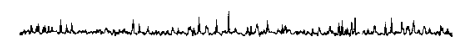

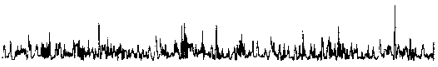

Figure 6. Effects of medial $(1.5 \mathrm{~mm})$ and lateral $(2 \mathrm{~mm})$ transverse (left) hemisections on DIG activity during RJMs. $A$ and $B$, DIG activity during RJMs induced by left $(A)$ or right $(B)$ cortical stimulation prior to hemisections. $C$ and $D$, DIG activity after another transection $(3.5 \mathrm{~mm}$ rostral to obex) extending only $1.5 \mathrm{~mm}$ lateral to midline. Note that, in $C$, during left cortical stimulation the right DIG exhibits oscillation, while the left DIG activity is almost abolished. In $D$, during stimulation of the right cortex, the amplitudes of both DIG are somewhat reduced, but a fundamental oscillation is still observed. $E$ and $F$, Data taken after another transection (same animal) that was still 3.5 $\mathrm{mm}$ rostral to the obex but now extended another $500 \mu \mathrm{m}$ lateral to the previous transection in $C$ and $D$. Note the abolition of only the left DIG in $E$ while, in $F$, rhythmical DIG activity was completely abolished. Time calibration, $F, 200$ msec. a selective transverse hemisection of this nucleus was performed. Figure 7 shows an example of RJMs and the jaw-opening response of the DIG EMG induced by lip stimulation (jawopening reflex) before (Fig. $7, A, B$ ) and after (Fig. 7, $C, D$ ) sensory nucleus hemisection. When the transection was sufficient to abolish the reflex response of the DIG (Fig. $7 D$ ), the RJMs induced by stimulation of the cortex were unaffected (Fig. $7 C$ ). This suggests that the abolishment of the RJMs and the reduction of the DIG response induced by stimulation of the right cortex after a hemisection of the brain stem core at the level of the rostral IO were most likely not due to interruption of fibers originating from interneurons within the spinal trigeminal nucleus.

In order to determine the importance of an intact midbrain RF on the initiation of RJMs, transverse hemisections of the left brain stem were made at a level just rostral to Mot V. The hemisections were made so as to spare only the pontine nuclei and intermingled corticobulbar fibers. The level of transection is shown in Figures $3 B$ and $9 G$. Such hemisections did not abolish the rhythmical, bilateral DIG EMG activity during RJMs induced by stimulation of the left or right cortex (Fig. 8). Furthermore, the CDs (Fig. $8 E$ ) and BDs (Fig. $8 F$ ) were not significantly affected. On the other hand, electrolytic lesions of the ventromedial quadrant of the brain stem just rostral to the Mot V (Fig. 9G, hatched area) were found to completely abolish both the SPT (not shown) and RJMs induced by stimulation of the left cortex (Fig. 9D), while leaving the responses to stimulation of the right cortex unaffected (Fig. $9 E$ ). It was also observed that stimulation of this brain stem area prior to lesioning was able to induce RJMs at relatively low intensities ( $<100 \mu \mathrm{A}$; Fig.
$9 C$ ). After lesioning, it was not possible to evoke RJMs by the brain stem stimulation (Fig. 9F). These data indicate that (1) the cortical pathways responsible for activation of RJMs traverse the ventral quadrant of the midbrain and are most likely direct corticobulbar fibers originating in the masticatory cortex that initially decussate at the level of Mot V, and (2) the midbrain RF is not essential for RJM production.

\section{Discussion}

The data of the present study have addressed a number of points with respect to the neural circuitry responsible for cortically induced RJMs. It was shown that (1) the area of neuraxis that must remain intact for cortically induced RJMs to occur is located between the Mot V and the rostral third of the IO of the medulla; (2) a component of the CPG(s) for RJMs and/or the output pre-motoneuronal cells of the CPG are most likely located in the parvocellular reticular formation between the trigeminal motor nucleus of the pons and the rostral level of the IO of the medulla; and (3) there exist neuronal oscillators in each side of the lower brain stem that are exclusively controlled by the contralateral masticatory cortex, and, as a result of this arrangement, each CPG residing in one side of the lower brain stem has the capability of producing bilateral coordination of jaw-opener motoneurons.

\section{Evidence that the bilateral coordination of jaw-opener motoneurons is controlled by each neuronal oscillator}

Stimulation of either left or right masticatory cortex produces bilateral, synchronous activation of both jaw-opener motoneuronal pools during RJMs (Kawamura and Tsukamoto, 1960a, 


\section{LEFT SENSORY NUCLEUS HEMISECTION}
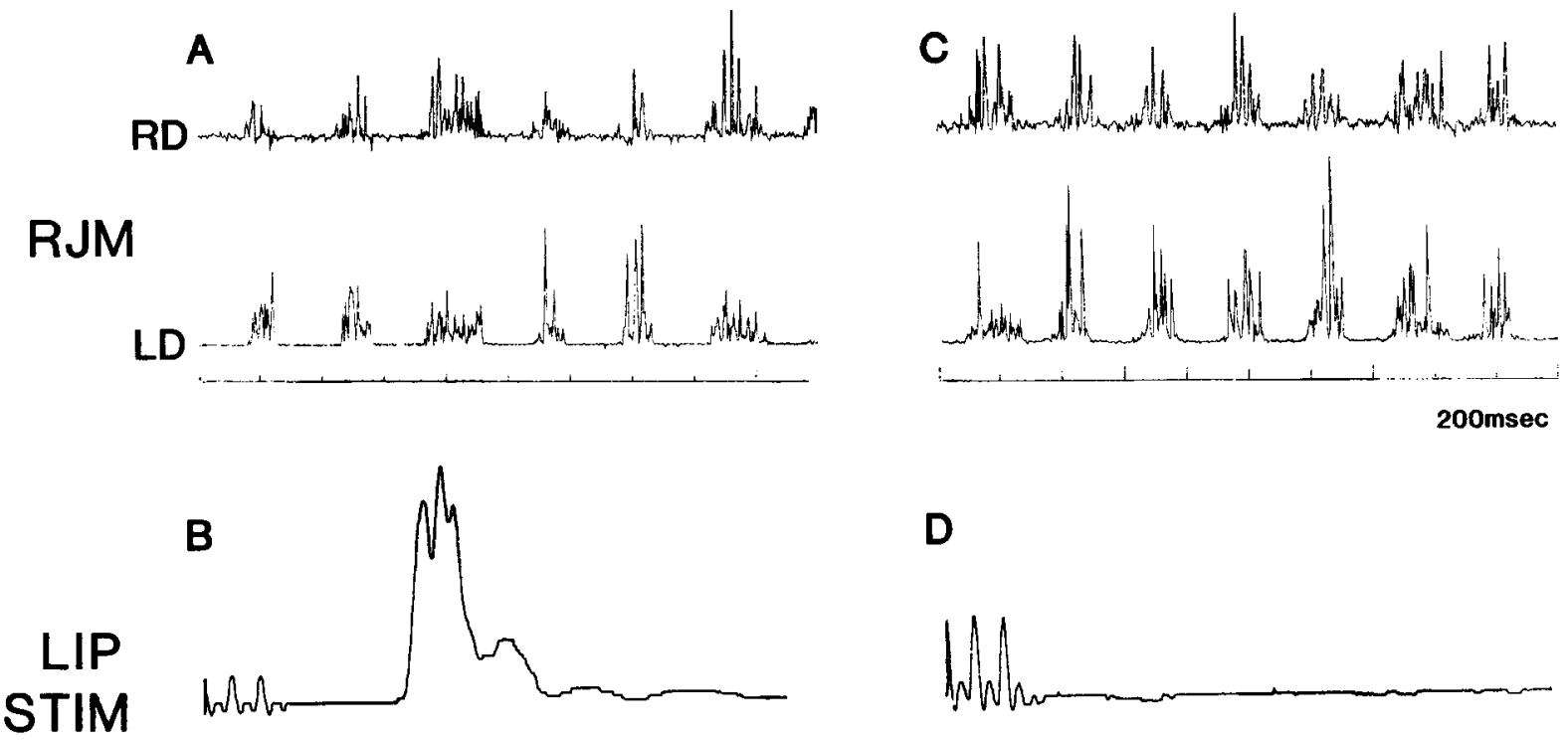

$4 \mathrm{msec}$

Figure 7. Effect of a discrete transverse hemisection of the left trigeminal spinal nucleus of $\mathrm{V}$ on rhythmical DIG activity and the jaw-opening reflex. $A$ and $B$, Control DIG responses to repetitive stimulation of the right cortex $(A)$ or SPT stimulus to the lip $-500 \mathrm{~Hz}, 3 \mathrm{pulses}, 0.2 \mathrm{msec}(B)$. $C$ and $D$, DIG responses after a discrete hemisection of the spinal nucleus at the level of the nucleus interpolaris. The transection started 2.5 mm lateral to the midline and extended another $1 \mathrm{~mm}$. Note that when the jaw-opening reflex was abolished by the transection $(D)$, rhythmical activity in both the left and right DIG EMG was still observed. Note the different time calibrations in $A$ and $C$ vs $B$ and $D$.

b; Lambert et al., 1985; Lund et al., 1984; see Dubner et al., 1978 , or Luschei and Goldberg, 1981, for review). One question that has not been resolved with respect to RIMs is how this coordination is produced. Since the rodent corticobulbar fibers project bilaterally (Zimmerman et al., 1964), the possibility exists that each cortex activates contra- and ipsilateral polysynaptic pathways to trigeminal pre-motoneurons. It has more recently been demonstrated with HRP retrograde labeling techniques that there are projections from both ipsi- and contralateral parvocellular RFs to a given trigeminal motor nucleus (Mizuno et al., 1983; Travers and Norgren, 1983; Vornov and Sutin, 1983). This anatomical arrangement suggests that a possible neuronal substrate for bilateral control of jaw opening during the RJM cycle could originate in pre-motoneurons located in a given side of the lower brain stem. Further support for this comes from electrophysiological experiments. Microstimulation of the bulbar reticular formation evokes bilateral short-latency activity in DIG motoneurons (Takatori et al., 1981). Contralateral cortical stimulation also produces short-latency EPSPs in these reticular neurons (Nakamura et al., 1979; Nozaki et al., 1983).

It has not, however, been demonstrated that a neuron within the medullary RF both projects to the ipsilateral trigeminal motor nucleus and receives convergent projections from both ipsilateral and contralateral masticatory cortex. In our experiments, selective midline transections of the lower brain stem were able to completely abolish both the short-latency jawopening response and rhythmical activity in the ipsilateral DIG muscle during either SPT or repetitive stimulation of the cortex. Hemisections of the lower brain stem at the IO completely abolished rhythmical activity in both ipsi- and contralateral motoneurons during stimulation of the cortex on the opposite side of the hemisection. During these conditions, stimulation of the cortex on the same side as the hemisection produced rhythmical activity in only the contralateral DIG motoneurons. This lack of activity in the ipsilateral DIG was not due to destruction of trigeminal motoneurons or fibers, since in all cases stimulation of intra-oral structures was capable of inducing the jaw-opening reflex. These observations were also not a function of cortical loci. The lack of activity was present regardless of electrode placement or intensity of stimulation. These data demonstrate that a given cortex activates neurons within the contralateral lower brain stem that then project to ipsilateral and/ or contralateral jaw-opener motoneurons. Consistent with these data are the observations that the short-latency bilateral DIG EMG responses produced by SPT cortical stimulation are approximately $2 \mathrm{msec}$ longer for the ipsilateral than for the contralateral DIG EMG (Lambert et al., 1985; Lund et al., 1984). This longer delay could be accounted for by a recrossing to the ipsilateral side.

Based on our present data, a schematic representation of the brain stem neuronal pathways and networks mediating cortically induced RJMs is shown in Figure 10. It is proposed that RJMs elicited by stimulation of a given side of cortex are produccd by sclcctive activation of corticobulbar fibers originating in the contralateral masticatory cortex, which decussate between Mot $\mathrm{V}$ and the IO. These fibers make synaptic contact with both pre-motoneuronal cells in the parvocellular RF and a CPG that contains at least some of its components in the parvocellular and possibly medial reticular formation. Activation of these premotoneurons and modulation of these pre-motoneurons by a CPG network(s) in the contralateral brain stem produce coordinated rhythmical activity in both left and right DIG muscles.

Location of $C P G(s)$ for RJMs

At present there have not been any detailed lesion or transection experiments defining the rostral-caudal or medial-lateral boundaries of the CPG network(s) for RJMs. Previous studies 


\section{CONTROL}

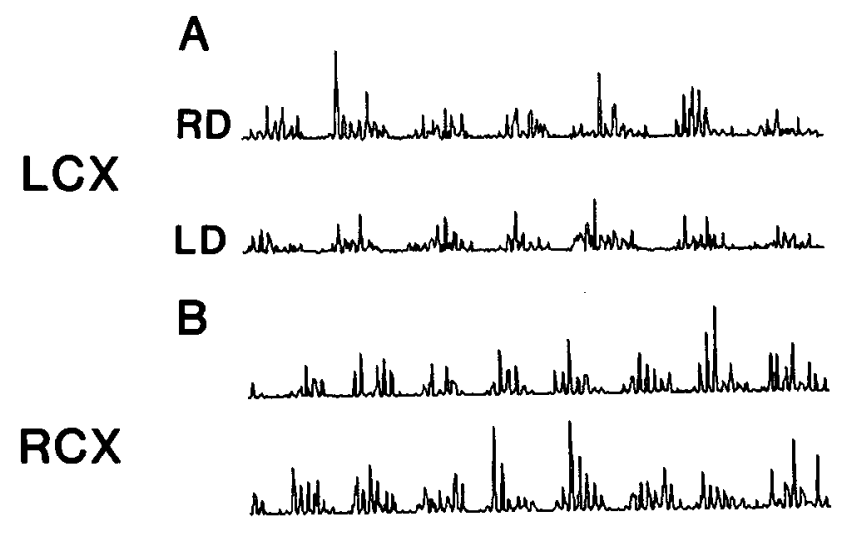

E

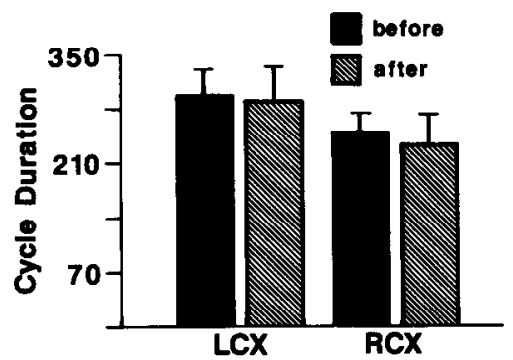

HEMISECTION -MIDBRAIN-

C

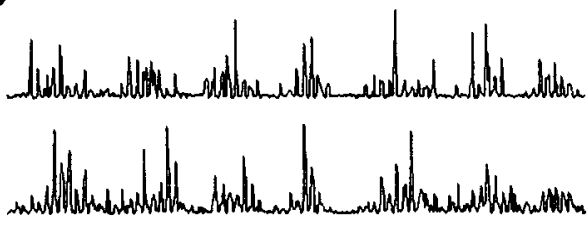

D

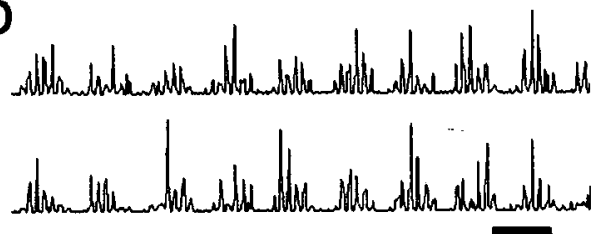

F

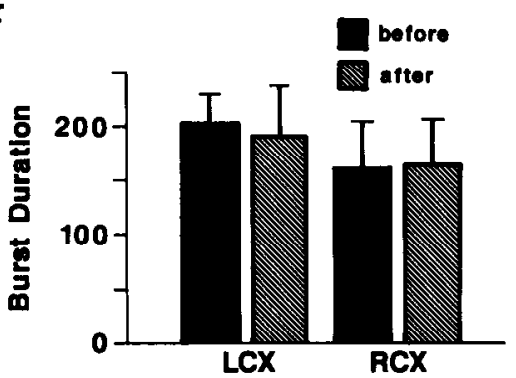

Figure 8. Effect of a left transverse hemisection of the caudal midbrain (sparing pyramidal tract) on rhythmical DIG EMG activity. $A$ and $B$, DIG EMG activity induced by left $(A)$ or right $(B)$ cortical stimulation prior to the hemisection. $C$ and $D$, EMG activity after a hemisection of the left midbrain approximately $500 \mu \mathrm{m}$ rostral to Mot V. The level of the transection is shown in Figure $3 B$ and $9 G$. Time calibration, $D, 200 \mathrm{msec}$. $E$ and $F$, Mean cycle $(E)$ and burst $(F)$ durations of the contralateral DIG EMG during stimulation of the left $(L C X)$ or right $(R C X)$ cortex before (black bars) and after (hatched bars) the hemisection. Vertical lines, Standard deviations. Student's $t$ test showed no statistical differences for CDs or BDs prior to or after the transection.

have shown that the networks responsible for RJMs must reside caudal to the rostral midbrain (Bremer, 1923; Grill and Norgren, 1978; Lund and Dellow, 1971; Thexton et al., 1980). Our experiments have defined these boundaries more precisely. With respect to a caudal boundary, serial transverse hemisections of the medulla starting at the obex demonstrated that the ability to induce RJMs by repetitive cortical stimulation and shortlatency activity in DIG motoneurons is critically dependent on the integrity of a region of medulla at the level of the rostral IO. Hemisections caudal to this level had minimal effects on the CDs or BDs of the digastric EMGs during RJMs (Table 1). This suggests that either the critical network(s) responsible for oscillation have some component at the level of and/or rostral to the IO, or that there exists a series of parallel, coupled oscillators distributed throughout the lower brain stem that require a critical mass of neurons for normal function. Our experiments cannot directly distinguish between the two possibilities, but if a series of distributed coupled oscillators does exist, they would each have to possess extremely strong interoscillator synaptic coupling themselves and very similar inherent oscillatory frequencies, such that any disruption in a part of the network would not affect the remaining oscillators' inherent output. If the system did not possess these properties, then one might expect that the output of the system would exhibit a change in the fundamental oscillatory frequency and a significant increase in variability when elements of the system were perturbed. In the present experiments, after either a bilateral transection or leftside hemisection of the brain stem, $C D$ and variability remained relatively constant prior to complete abolition of RJMs (Fig. $5 F$; Table 1). The observed reduction in amplitude of the DIG
EMG prior to abolition of RJMs most probably resulted from a transection of some of the corticobulbar fibers or the fibers of the pre-motoneurons, which have been shown anatomically to be distributed throughout the lateral RF of the lower brain stem (Mizuno et al., 1983; Travers and Norgren, 1983; Vornov and Sutin, 1983).

It could be argued that the lateral extent of our hemisections interrupted trigeminal fibers originating in the spinal trigeminal nucleus that are involved with mediating reflcx jaw-opening (Sumino, 1971). Although it has been demonstrated that phasic peripheral reflex activation is not responsible for the genesis of the oscillation (Chandler and Goldberg, 1982; Dellow and Lund, 1971; Nakamura et al., 1976), cortical projections to the spinal trigeminal nucleus are abundant in various animal spccics (Brodal et al., 1956; Zimmerman et al., 1964) and, therefore, the possibility exists that these neurons could be a source of excitation to jaw-opening motoneurons during cortically induced RJMs. In the present experiments, selective hemisection of the spinal trigeminal nucleus, which abolished the jaw-opening reflex, had no effect on the cortically induced RJMs (Fig. 7). Therefore, the CPG network(s) and/or the output interneurons of the CPG most likely have a lateral border in the lateral (parvocellular) RF.

Although the precise nature of the elements comprising the CPG is not known, both anatomical evidence from HRP injections into the trigeminal motor nucleus (Mizuno et al., 1983; Travers and Norgren, 1983; Vornov and Sutin, 1983) and single-unit recordings during either SPT stimulation of the masticatory cortex (Nozaki et al., 1983) or during RJMs in the cat (Nakamura et al., 1980, 1982b) suggest that it is located in the 


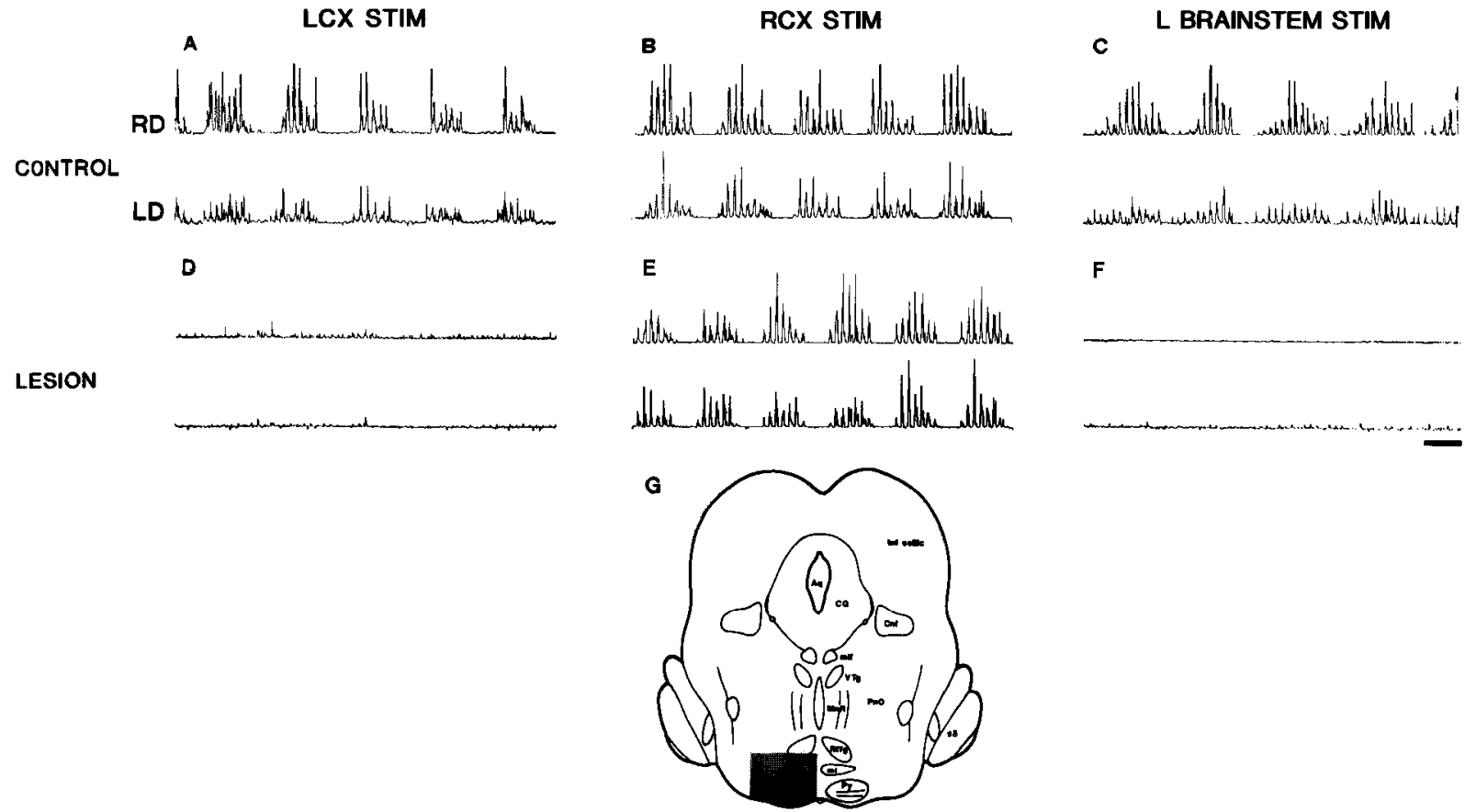

Figure 9. Effect of an electrolytic lesion of the ventromedial midbrain on rhythmical DIG EMG activity. $A$ and $B$, EMG activity induced by left $(A)$ or right $(B)$ cortical stimulation prior to the lesion. $C$, EMG activity induced by left brain stem stimulation (depicted by hatched region in $G$ ). $D-F$, EMG activity after an electrolytic lesion $-500 \mu \mathrm{A}$ (anodal), $60 \mathrm{sec}$. Note that rhythmical activity induced by stimulation of left cortex ( $D$ ) or left brain stem $(F)$ was abolished, while rhythmical activity induced by the right cortex $(E)$ was maintained. $G$, Transverse section of the caudal midbrain where the lesion was located (hatched region). The lesion extended approximately $\pm 500 \mu \mathrm{m}$ from this region. Hemisections that spared this region had minimal effect on rhythmical DIG activity (see Fig. 8). Abbreviations: $A q$, Cerebral aqueduct; $C G$, central gray; $C n f$, cuneiform nucleus; $m l f$, medial longitudinal fasciculus; $M n R$, median raphe nucleus; Inf collic, inferior colliculus; $m l$, medial lemniscus; $P n O$, pontine reticular nucleus oralis; $R t T g$, reticulotegmental nucleus; $s 5$, sensory root of trigeminal nerve; $V T g$, ventral tegmental nucleus.

pontine and medullary RF. The HRP experiments have shown widespread retrograde labeling of cells spanning the parvocellular RF of the pons and medulla (Mizuno et al., 1983; Travers and Norgren, 1983; Vornov and Sutin, 1983) without labeling in the medial or gigantocellular portion of the RF. It has also been shown that preterminal degeneration of corticobulbar fibers occurs with highest density in the parvocellular, as opposed to the gigantocellular, RF (Zimmerman et al., 1964). This supports our findings that the more lateral transections abolished the short-latency DIG response to SPT and repetitive stimulation of the contralateral cortex. Electrophysiological experiments have shown that cells of the bulbar RF can be activated antidromically from stimulation of the trigeminal motor nucleus and orthodromically by stimulation of the masticatory cortex (Nozaki et al., 1983). It is not exactly clear what percentage of cells were localized to parvocellular, as opposed to gigantocellular, RF. It has also been shown in the cat that transverse hemisections of the medial RF at the level of the rostral IO are not able to abolish the short-latency jaw-opening response to cortical stimulation (see plate 1 in Nakamura et al., 1982a). When the transection was made at a level just rostral to the facial nucleus at the level of the superior olivary nucleus and including the lateral RF, the short-latency jaw-opening response was abolished (Nakamura et al., 1982a). Although RJMs were not examined, those data are consistent with our findings that only hemisections that included the parvocellular RF were effective in abolishing the oscillatory component of RJMs and the short-latency DIG response to cortical stimulation. Our data, demonstrating that the critical transection for RJM abolition occurred at the rostral IO, differ from those of others (Nakamura et al., 1982a) who found the critical transection to be rostral to the facial nucleus. In those experiments, the transections at the level of the rostral IO did not include the lateral RF (see plate
1, Nakamura et al., 1982a). This could account for the observed differences

The rostral boundary of the CPG network(s) appears to be located at the level of the trigeminal motor nucleus. Serial transverse hemisections starting at the rostral midbrain and placed as little as $500 \mu \mathrm{m}$ rostral to $\mathrm{Mot} V$, which spared the ventralmedial brain stem but eliminated the core of the RF of the midbrain and rostral pons, had minimal effects on RJMs induced by stimulation of either cortex (Fig. 8). Hemisections placed between the Mot V and IO abolished RJMs induced by either cortex. On the other hand, electrolytic lesions of the ventral quadrant rostral to the Mot V, which included the pontine nuclei and the corticobulbar fibers but spared the RF of the rostral pons and midbrain, completely abolished RJMs induced by stimulation of only the ipsilateral cortex (Fig. 9). This latter effect cannot be due to interruption of pontocerebellar fibers, since in our preparations the cerebellum was ablated and RJMs were easily elicited. Based on preparations that have undergone decerebrations at the rostral midbrain, previous investigators have suggested that the CPG resides between rostral midbrain and medulla (Bremer, 1923; Lund and Dellow, 1971; Thexton et al., 1980). None of those studies carefully examined RJM cycle characteristics, and furthermore, in some of them, only fragmented jaw movements were shown. Our data demonstrate that the oscillatory network(s) is not dependent on an intact midbrain RF, and most likely its rostral boundary is at the level of, or caudal to, Mot V. The paucity of retrograde labeling of cells in the midbrain from Mot V injections of HRP supports our data, at least with respect to pre-motoneuronal cells involved in mediating the short-latency jaw-opening response to cortical stimulation. It could be argued that the oscillatory signal arises in the cortex and is relayed to pre-motoneurons of the lower brain stem. This is unlikely, since we and others (Lund 
and Dellow, 1971; Tal and Chandler, unpublished observations) have observed RJMs induced by stimulation of the pyramidal tract at a level just rostral to the trigeminal motor nucleus in decerebrate preparations. Furthermore, single-cell recording from the cortex during chewing has revealed few cells rhythmically active during a particular phase of the chewing cycle (Hoffman and Luschei, 1980; Lund and Lamarre, 1974). Therefore, our data suggest that, as long as the corticobulbar command system remains intact, the minimum circuitry required for cortically induced oscillation of the jaw to occur resides between Mot $V$ and the rostral IO.

\section{Evidence for the existence of oscillatory circuitry in each} side of the lower brain stem

There has been no direct evidence in the jaw system to suggest that a given side of the brain stem has the capability of producing oscillation independent of the integrity of contralateral side. Sumi (1971) has shown that midline transections extending from the corpus callosum to the rostral border of the superior colliculus have no effect on cortically induced RJMs. When lesions were placed more caudally in the midbrain, RJMs induced by cortical stimulation were abolished. In those experiments the differentiation between ipsi- and contralateral DIG EMG activity was not made, and the precise level of the midline transection was not described. The data presented from our experiments show that, for oscillation to occur in any DIG muscle by stimulation of the cortex, the integrity of the contralateral lower brain stem is critical. Selective anatomical uncoupling of the lower brain stem through midline transections abolished activity induced by either SPT or repetitive stimulation of the cortex in the ipsilateral DIG EMG while leaving the contralateral DIG EMG unaffected (Figs. 1, 2). Midline transections between the Mot $\mathrm{V}$ and the facial nucleus completely abolished rhythmical bilateral DIG activity induced by stimulation of either cortex, while midline transections rostral to Mot $V$ had no effect on RJMs induced by stimulation of either cortex (Fig. 4). This suggests that the corticobulbar fibers originating in the masticatory cortex cross to the contralateral brain stem at the level of and caudal to the Mot V nucleus.

Hemisections of the lower brain stem at the level of the rostral IO completely abolished rhythmic EMG activity and the shortlatency responses in both left and right DIG muscles induced by stimulation of the cortex on the opposite side of the hemisection (Fig. 5). Under these conditions, stimulation of the cortex on the same side as the hemisection produced rhythmical activity only in the contralateral DIG EMG. Furthermore, the cycle characteristics of the unaffected DIG EMG were relatively unchanged (Fig. 5, Table 1). The most parsimonious interpretation of these findings would be that a given cortex initiates activity in only the contralateral brain stem, which in itself contains circuitries capable of producing rhythmical activity in both left and right DIG motoneurons. Most models of a CPG for RJMs have not addressed the question of the existence of bilateral neuronal oscillators (Chandler and Goldberg, 1982; Murakami et al., 1980; Nakamura et al., 1979; Sumi, 1971). Independent CPGs on each side of the spinal cord that control the muscles about a given joint have been suggested for locomotion (Grillner, 1981) and have been demonstrated in the respiratory system by anatomical uncoupling of both sides of the lower brain stem (Gromysz and Karczewski, 1981a, b).

Our results indicate the possibility of an independent oscillator(s) within each side of the lower brain stem that is exclusively controlled by the contralateral cortex and that can coordinate DIG motoneurons bilaterally. This apparent redundancy in neuronal oscillators is important when one considers that each cortex initiates not only rhythmical opening and closing activity, but also has the capability of initiating a specific lateral component to the movement sequence (Lambert et al., 1985).

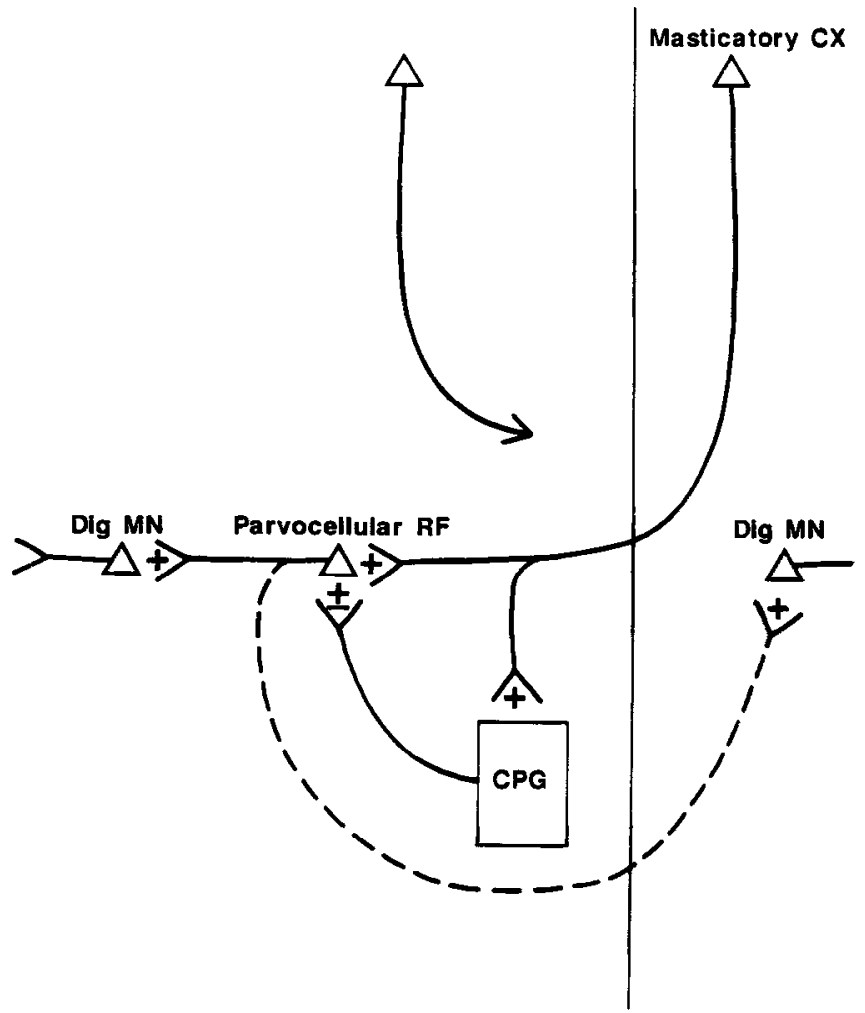

Figure 10. Schematic diagram of proposed pathways and area of lower brain stem that mediate cortically induced RJMs. Dotted lines, Possible polysynaptic connections. Each side of cortex activates a CPG located in the contralateral brain stem, which then alternately facilitates and inhibits the DIG pre-motoneurons located in the parvocellular $R F$. Bilateral activation of DIG motoneurons occurs through activation of DIG pre-motoneurons in the contralateral brain stem that have projections to both DIG motoneuronal pools. The minimal neural circuitry required for oscillation of the jaw resides between the Mot $\mathrm{V}$ and the rostral third of the IO and nucleus ambiguus. The precise location and anatomical components of the $C P G$ are unknown. An identical circuit in the other side of the brain stem would be activated by stimulation of the left cortex.

With this type of neuronal constraint each brain-stem CPG would then be responsible for integrating the fundamental masticatory oscillation with a specific vertical and lateral muscle synergy to produce the specific movement trajectory during mastication.

\section{References}

Bremer, F. (1923) Physiologie nerveuse de la mastication chez le chat et le lapin. Reflexes de mastication. Reponses masticatrices corticales et centre cortical du gout. Arch. Int. Physiol. 21: 308-352.

Brodal, A., T. Szabo, and A. Torvik (1956) Corticofugal fibers to sensory trigeminal nuclei and nucleus of solitary tract: An experimental study in the cat. J. Comp. Neurol. 106: 527-555.

Chandler, S. H., and L. J. Goldberg (1982) Intracellular analysis of synaptic mechanisms controlling spontaneous and cortically induced rhythmical jaw movements in the guinea pig. J. Neurophysiol. 48: 126-138.

Daniel, W. W. (1983) Biostatistics: A Foundation for Analysis in the Health Sciences, 3rd Ed., Wiley, New York.

Dellow, P. G., and J. P. Lund (1971) Evidence for central timing of rhythmical mastication. J. Physiol. (Lond.) 215: 1-13.

Dubner, R., B. J. Sessle, and A. T. Storey (1978) Neural Basis for Oral and Facial Function, Plenum, New York.

Goldberg, L. J., S. H. Chandler, and M. Tal (1982) Relationship between jaw movements and trigeminal motoneuron membrane-potential fluctuations during cortically induced rhythmic jaw movements in the guinea pig. J. Neurophysiol. 48: 110-125. 
Goodwin, G. M., and E. S. Luschei (1974) Effects of destroying spindle afferents from jaw muscles on mastication in monkeys. J. Neurophysiol. 37: 967-981.

Grill, H. J., and R. Norgren (1978) The taste reactivity test. II. Mimetic responses to gustatory stimuli in chronic thalamic and chronic decerebrate rats. Brain Res. 143: 281-297.

Grillner, S. (1981) Control of locomotion in bipeds, tetrapods, and fish. In Handbook of Physiology, Sect. 1: The Nervous System, Vol. 2, J. M. Brookhart and V. B. Mountcastle, eds., pp. 1174-1236, Williams \& Wilkins, Baltimore, MD.

Gromysz, H., and W. A. Karczewski (1981a) The effects of brainstem transection on respiratory activity in the rabbit. Acta Neurobiol. Exp. 41: 225-235.

Gromysz, H., and W. A. Karczewski (1981b) Respiratory activity generated by a split brainstem preparation in the rabbit. Acta Neurobiol. Exp. 41: 237-242.

Hoffman, D. S., and E. S. Luschei (1980) Responses of monkey precentral cortical cells during a controlled jaw bite task. J. Neurophysiol. 44: 333-348.

Kawamura, Y., and S. Tsukamoto (1960a) Analysis of jaw movements from the cortical jaw motor area and amygdala. Jpn. J. Physiol. 10: $471-488$.

Kawamura, Y., and S. Tsukamoto (1960b) Neural descending pathways from the cortical jaw motor area and amygdaloid nucleus to jaw muscles. Jpn. J. Physiol. 10: 489-498.

Kuypers, H. G. J. M. (1958) An anatomical analysis of cortico-bulbar connexions to the pons and lower brain stem in the cat. J. Anat. 92: 193-218.

Lambert, R. W., L. J. Goldberg, and S. H. Chandler (1985) The relationship between cortically induced mandibular movements and lateral pterygoid and digastric muscle EMG activity in the anesthetized guinea pig. Brain Res. 329: 7-17.

Lund, J. P., and P. G. Dellow (1971) The influence of interactive stimuli on the rhythmical masticatory movements in rabbits. Arch. Oral Biol. 16: 215-223.

Lund, J. P., and Y. Lamarre (1974) Activity of neurons in the lower precentral cortex during voluntary and rhythmical jaw movements in the monkey. Exp. Brain Res. 19: 282-299.

Lund, J. P., and K. Olsson (1983) The importance of reflexes and their control during jaw movements. Trends Neurosci. 6: 458-463.

Lund, J. P., S. Rossignol, and T. Murakami (1981) Interactions between the jaw-opening reflex and mastication. Can. J. Physiol. 59: 683-690.

Lund, J. P., K. Sasamoto, T. Murakami, and K. A. Olsson (1984) Analysis of rhythmical jaw movements produced by electrical stimulation of motor-sensory cortex of rabbits. J. Neurophysiol. 52: 1014 1029.

Luschei, E. S., and L. J. Goldberg (1981) Neural mechanisms of mandibular control. In Handbook of Physiology, Vol. 2: Motor Control, Pt. 2, J. M. Brookhart, V. B. Mountcastle, V. B. Brooks, and S. R. Geiger, eds., pp. 1237-1274, American Physiological Society, Bethesda, MD.

Mizuno, N., Y. Yasui, S. Nomura, K. Itoh, A. Konishi, M. Takada, and M. Kudo (1983) A light and electron microscope study of premotor neurons for the trigeminal motor nucleus. J. Comp. Neurol. 215: $290-298$.
Murakami, T., J. P. Lund, and M. Beaulieu (1980) Elaboration and modification of patterns of rhythmical mastication. In Jaw Move ment, K. Kubota, Y. Nakamura, and G. H. Schumacher, eds., pp. 305-308, VEB Verlag Volk und Gesundheit, Berlin.

Nakamura, Y., Y. Kubo, S. Nozaki, and M. Takatori (1976) Cortically induced masticatory rhythm and its modification by tonic peripheral inputs in immobilized cats. Bull. Tokyo Med. Dent. Univ. 23: 101107.

Nakamura, Y., M. Takatori, Y. Kubo, S. Nozaki, and S. Enomoto (1979) Masticatory rhythm formation-facts and a hypothesis. In Integrative Control Functions of the Brain, Vol. 2, N. Tsukahara, K. Kubots, and K. Yagi, eds., pp. 321-331, Kodansh Scientific, Tokyo.

Nakamura, Y., S. Enomoto, and M. Katoh (1980) The role of medial bulbar reticular neurons in the orbital cortically induced masticatory rhythm in cats. Brain Res. 202: 207-212.

Nakamura, Y., M. Katoh, S. Enomoto, and K. Hiraba (1982a) Neural pathway involved in the excitation of motoneurones to jaw-opening muscles by stimulation of the orbital cerebral cortex in the cat. Arch. Oral Biol. 27: 283-287.

Nakamura, Y., K. Hiraba, S. Enomoto, and Y. Sahara (1982b) Bulbar reticular unit activity during food ingestion in the cat. Brain Res. 253. 312-316.

Nakamura, Y., K. Hiraba, M. Taira, Y. Sahara, S. Enomoto, M. Katoh, and A. Iriki (1984) Activity during active sleep of bulbar reticular neurons firing rhythmically during mastication in cats. Exp. Neurol. 85: $178-186$

Nozaki, S., S. Enomoto, and Y. Nakamura (1983) Identification and input-output properties of bulbar reticular neurons involved in the cerebral cortical control of trigeminal motoneurons in cats. Exp. Brain Res. 49: 363-372.

Sumi, T. (1971) Modification of cortically evoked rhythmic chewing and swallowing from midbrain and pons. Jpn. J. Physiol. 21: 489 506.

Sumi, T. (1977) Interrelation between rhythmic mastication and reflex deglutition as studied on the unitary activity trigeminal motoneurons in rabbits. Jpn. J. Physiol. 27: 687-699.

Sumino, R. (1971) Central neural pathways involved in the jaw-opening reflex in the cat. In Oral-Facial Sensory and Motor Mechanisms, R. Dubner and Y. Kawamura, eds., pp. 315-329, Appleton-CenturyCrofts, New York.

Takatori, M., S. Nozaki, and Y. Nakamura (1981) Control of trigeminal motoneurons exerted from bulbar reticular formation in the cat. Exp. Neurol. 72: 122-140.

Thexton, A. J., C. Griffiths, and J. McGarrick (1980) Evidence for peripheral activation of the trigeminal rhythm generator in the decerebrate rat, obtained by Fourier analysis of conditioning testing curves. Arch. Oral Biol. 25: 491-494.

Travers, J. B, and R. Norgren (1983) Afferent projections to the oral motor nuclei in the rat. J. Comp. Neurol. 220: 280-298.

Vornov, J. J., and J. Sutin (1983) Brainstem projections to the normal and noradrenergically hyperinnervated trigeminal motor nucleus. J. Comp. Neurol. 214: 198-208.

Zimmerman, E. A., W. W. Chambers, and C. N. Liu (1964) An experimental study of the anatomical organization of the cortico-bulbar system in the albino rat. J. Comp. Neurol. 123: 301-324. 